\title{
Fate of Tetracapsuloides bryosalmonae (Myxozoa) after infection of brown trout Salmo trutta and rainbow trout Oncorhynchus mykiss
}

\author{
Gokhlesh Kumar, Ahmed Abd-Elfattah, Mona Saleh, Mansour El-Matbouli* \\ Clinical Division of Fish Medicine, Department for Farm Animals and Veterinary Public Health, \\ University of Veterinary Medicine, Vienna, Austria
}

\begin{abstract}
Tetracapsuloides bryosalmonae (Myxozoa) is the causative agent of proliferative kidney disease in salmonids. We assessed differences in intensity of $T$. bryosalmonae infection between brown trout Salmo trutta and rainbow trout Oncorhynchus mykiss from the clinical phase of infection onwards. Specific pathogen-free fish were exposed to T. bryosalmonae spores under controlled laboratory conditions and sampled at 6, 8, 10, 12, 14, and 17 wk post exposure (wpe), and the transmission of $T$. bryosalmonae from infected fish to the bryozoan Fredericella sultana was observed. Parasite load was determined in fish kidneys by quantitative real-time PCR (qRTPCR), and parasite stages were detected in kidney, liver, and spleen tissues at different time points by immunohistochemistry. $T$. bryosalmonae was successfully transmitted from infected brown trout to F. sultana colonies but not from infected rainbow trout. Body length and weight of infected brown trout did not differ significantly from control brown trout during all time points, while length and weight of infected rainbow trout differed significantly compared to controls from 10 to 17 wpe. qRT-PCR revealed that parasite load was significantly higher in kidneys of brown trout compared with rainbow trout. Immunohistochemistry showed high numbers of intra-luminal stages (sporogonic stages) in kidneys of brown trout with low numbers of pre-sporogonic stages. Sporogonic stages were not seen in kidneys of rainbow trout; only high numbers of pre-sporogonic stages were detected. Numbers of pre-sporogonic stages were low in the spleen and liver of brown trout but high in rainbow trout. These data confirmed that there are differences in the development and infection progress of $T$. bryosalmonae between brown trout and rainbow trout.
\end{abstract}

KEY WORDS: Myxosporean parasite $\cdot$ Immunohistochemistry $\cdot$ Proliferative kidney disease $\cdot$ PKD · Pre-sporogonic stages $\cdot$ Sporogonic stages

\section{INTRODUCTION}

Proliferative kidney disease (PKD) significantly affects both farmed and wild salmonid fish in Europe and North America, causes economic losses, and endangers wild fish populations (Feist et al. 2001, ElMatbouli \& Hoffmann 2002, Okamura et al. 2011). PKD is caused by the myxozoan parasite Tetracapsuloides bryosalmonae. Spores develop in the kidney tubules of infected fish and are released via urine to infect freshwater bryozoans (Morris \& Adams 2006, Grabner \& El-Matbouli 2008). Proliferation of T. bryo- salmonae induces a granulomatous cellular response in the interstitial tissue that induces swelling of the spleen and kidney (Ferguson \& Needham 1978, Clifton-Hadley et al. 1987).

Genetic differences between isolates of Tetracapsuloides bryosalmonae from North America and Europe have been recorded (Henderson \& Okamura 2004). Tops et al. (2004) did not observe infection of bryozoans after exposure to $T$. bryosalmonae-infected brown trout Salmo trutta, rainbow trout Oncorhynchus mykiss, or Chinook salmon O. tshawytscha. Later work showed that $T$. bryosalmonae-infected 
brown trout can release spores that are infective to bryozoans (Morris \& Adams 2006); however, rainbow trout infected with the European isolate of T. bryosalmonae were not able to infect bryozoans (Grabner \& El-Matbouli 2008).

El-Matbouli et al. (2009) showed no significant differences in the susceptibility of German (Hofer strain) and North American (Troutlodge) rainbow trout strains to Tetracapsuloides bryosalmonae with regard to clinical signs, mortality rates, and number of parasitic stages in kidney and spleen 4 to $16 \mathrm{wk}$ post exposure (wpe). Temperature- and time-related changes of $T$. bryosalmonae have been examined in the kidneys of rainbow trout from initial infection to the clinical phase of the disease (Bettge et al. 2009a,b). Further, those authors investigated temperature- and timerelated parasite distribution in the kidneys of rainbow trout from 47 to $201 \mathrm{~d}$ post exposure (dpe) at 12 and $18^{\circ} \mathrm{C}$ and suggested that parasite development and renal pathological lesions are temperature dependent (Schmidt-Posthaus et al. 2012). The chronological development of $T$. bryosalmonae has been well documented in rainbow trout (Kent \& Hedrick 1986, Bucke et al. 1991, Morris et al. 2005, Bettge et al. 2009b, Okamura et al. 2011, Schmidt-Posthaus et al. 2012), but little information is available on its development in brown trout (Clifton-Hadley \& Feist 1989, Grabner \& El-Matbouli 2009). All studies were based on exposure of fish to natural $T$. bryosalmonae-infected river waters and mainly focused on renal pathology.

Our objective was to compare the chronological development and intensity of infection of Tetracapsuloides bryosalmonae in kidney, spleen, and liver tissues of brown trout and rainbow trout from the clinical phase of infection onwards and transmission of $T$. bryosalmonae from infected fish to the bryozoan Fredericella sultana. Brown trout and rainbow trout were infected with spores of T. bryosalmonae, and the relative parasite load of kidneys was assessed by quantitative real-time PCR (qRT-PCR). The parasite stages were visualized and quantified in kidney, liver, and spleen tissues at different time points using immunohistochemistry, and transmission of T. bryosalmonae from infected fish to $F$. sultana was examined.

\section{MATERIALS AND METHODS}

\section{Fish}

Specific pathogen-free (SPF) brown trout and rainbow trout sac fry were obtained from a local hatchery and grown in the aquarium facility at the Clinical
Division of Fish Medicine, University of Veterinary Medicine, Vienna, Austria. Prior to infection, 10 brown trout and 10 rainbow trout were sampled randomly and tested for the presence of Tetracapsuloides bryosalmonae by qRT-PCR.

\section{Collection and preparation of Tetracapsuloides bryosalmonae spores}

Colonies of Fredericella sultana infected with Tetracapsuloides bryosalmonae were kept in a laboratory culture system according to Kumar et al. (2013). Infected colonies with zooids that contained mature spore sacs were dissected using fine needles and forceps on a microscope slide. The spores were washed with a small amount of tap water into a small plastic Petri plate.

\section{Experimental infection of brown trout and rainbow trout}

Sixty brown trout and 60 rainbow trout (mean length $5.5 \pm 0.5 \mathrm{~cm}$, mean weight $2.3 \pm 0.5 \mathrm{~g}$ ) were placed in flow-through aquaria. Prior to infection, the water supply to each aquarium was stopped and the volume reduced to $4 \mathrm{l}$. Free Tetracapsuloides bryosalmonae spores in suspension, released from 12 mature sacs, were added to all aquaria, which were then maintained with vigorous aeration for $24 \mathrm{~h}$ at $16.5 \pm 1^{\circ} \mathrm{C}$. Flow-through water was then restarted, and fish were maintained at $16.5 \pm 1{ }^{\circ} \mathrm{C}$. Additionally, 30 brown trout and 30 rainbow trout were held as non-infected controls for comparison of behavior, clinical signs, and qRT-PCR and immunohistochemistry analyses.

Ten fish from each exposure group and 5 control fish from each group were sampled at $6,8,10,12,14$, and 17 wpe. Fish were euthanized with an overdose of MS-222 anesthetic, and fish lengths and weights were measured. Kidney, spleen, and liver were sampled from all fish. Tissues were divided into 2 portions, 1 fixed in 10\% neutral buffered formalin for histology, and 1 in RNAlater (Qiagen) for molecular work.

\section{Cohabitation of SPF bryozoa with infected fish}

SPF Fredericella sultana colonies were cohabitated with infected brown trout and rainbow trout per the methods of Kumar et al. (2013). Briefly, at 8 wpe, 4 infected fish of each species were cohabitated for $8 \mathrm{~h} \mathrm{~d}^{-1}$ 
for $14 \mathrm{~d}$ with laboratory-hatched SPF F. sultana. After cohabitation, fish were transferred to an aquarium with spring water and fed. The bryozoan colonies were fed with algal species and observed daily using a dissecting microscope, up to 4 mo post-cohabitation.

\section{DNA extraction and qRT-PCR}

Kidneys were homogenized using a Tissue Lyser II (Qiagen), and DNA samples were extracted with a DNeasy blood and tissue kit (Qiagen) according to the manufacturer's instructions. DNA concentration was measured using a BioPhotometer (Eppendorf). qRT-PCR was performed according to the method described by Grabner \& El-Matbouli (2009) with minor modifications. Briefly, PKD-real primers (5'TGT CGA TTG GAC ACT GCA TG-3' and 5'-ACG TCC GCA AAC TTA CAG CT-3') were used to quantify parasite load, alongside trout insulin growth factor I (IGF I) primers used as a reference to normalize the amount of host tissue. The $166 \mathrm{bp} 18 \mathrm{~S}$ rDNA gene of Tetracapsuloides bryosalmonae and $67 \mathrm{bp}$ trout IGF I gene were cloned into a pDrive cloning vector using a PCR cloning kit (Qiagen). Plasmid DNA was purified using a QIAprep Miniprep kit (Qiagen) and digested with PstI restriction enzyme (Fermentas) to linearized plasmid DNA, and then its concentration was measured using a BioPhotometer. Ten-fold serial dilutions of linearized plasmid DNA were prepared in EB buffer (Qiagen). qRT-PCR reactions comprised $2 \times$ iQ SYBR Green Supermix (BIO-RAD), $0.4 \mu \mathrm{M}$ of each primer, and DNA template from brown trout and rainbow trout at different time points in a $25 \mu \mathrm{l}$ reaction volume. All samples were run in duplicate. Reactions with the PKD-real and IGF-I primers were run together on the same plate using CFX96 Touch Real-Time PCR detection system (BIO-RAD). Normalized quantities of parasite at different time points were $\log _{10}$-transformed and statistically analyzed.

\section{Histology and immunohistochemistry}

Tissue samples (kidney, spleen, and liver) were fixed in $10 \%$ neutral buffered formalin for $24 \mathrm{~h}$ and then washed, dehydrated, and embedded in paraffin wax. Sections were cut at $5 \mu \mathrm{m}$ and either stained with hematoxylin and eosin (H\&E) or processed for immunohistochemistry. Tetracapsuloides bryosalmonae monoclonal antibody P01 (Aquatic Diagnostics) was used according to the manufacturer's instructions. Antibody-antigen reaction was visualized using a Dako EnVision+ System-HRP (AEC) kit. Sections were counterstained with hematoxylin and examined for parasite stages. The number of parasite stages in each fish kidney section was counted as described by Schmidt-Posthaus et al. (2012).

\section{Statistical analysis}

Length, weight, parasite numbers, and $\log _{10}$ relative parasite load of kidneys from brown trout and rainbow trout were analyzed using linear mixed effect models. The differences between groups at each time point were analyzed using $t$-tests with Bonferroni correction alpha adjustment. For all statistical tests, a p-value $<0.05$ was regarded as significant. All statistical analyses were conducted with SPSS v20 software.

\section{RESULTS}

\section{Fish infection}

No mortalities occurred during initial exposure, and only 4 fish ( 3 brown trout and 1 rainbow trout) died during the experiment, most of them at 11 to 12 wpe. Lengths and weights of infected brown trout did not differ significantly from control brown trout (Figs. 1A \& 2A). Lengths of infected rainbow trout did not differ significantly from control rainbow trout at 6 to 10 wpe but differed significantly $(\mathrm{p}<0.01)$ at 12 to 17 wpe (Fig. 1B). Weight of infected rainbow trout did not differ significantly from controls at 6 to 8 wpe but differed significantly ( $p<0.01$ or $p<0.001)$ at 10 to 17 wpe (Fig. 2B).

Internal PKD signs were observed in the fish dissected at 8 wpe. Obvious internal signs of disease (renal hypertrophy, splenomegaly, and pale liver) were observed in brown trout at 10 wpe (Fig. 3) and in rainbow trout at 8 to 12 wpe. Moderate renal hypertrophy and splenomegaly were observed in brown trout at 8 and 12 to 17 wpe and in rainbow trout at 14 wpe. However, at 17 wpe, kidneys were normal in rainbow trout.

\section{Cohabitation of Fredericella sultana colonies with infected brown trout and rainbow trout}

Free-swirling spores and mature sacs were observed in the body cavities of Fredericella sultana colonies 4 wpe to infected brown trout (Fig. 4). In contrast, 


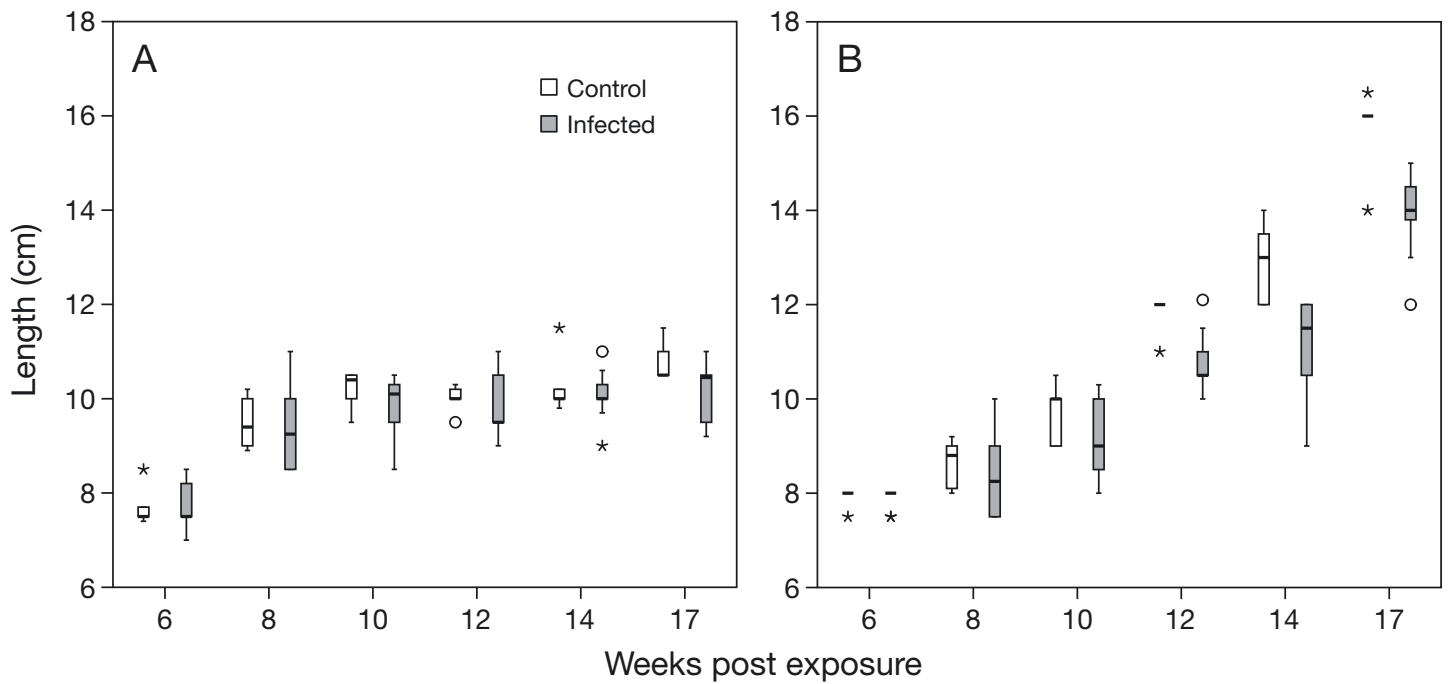

Fig. 1. Tetracapsuloides bryosalmonae infecting Salmo trutta and Oncorhynchus mykiss. Comparison of body length of infected and control fish at different time points: (A) brown trout, (B) rainbow trout. Box plots show the median value (horizontal lines), 25 and $75 \%$ quantiles (box), minimum and maximum (whiskers), and outliers (0). Stars indicate values which lie more than 3 times the interquartile range above (below) the $75 \%(25 \%)$ quantile

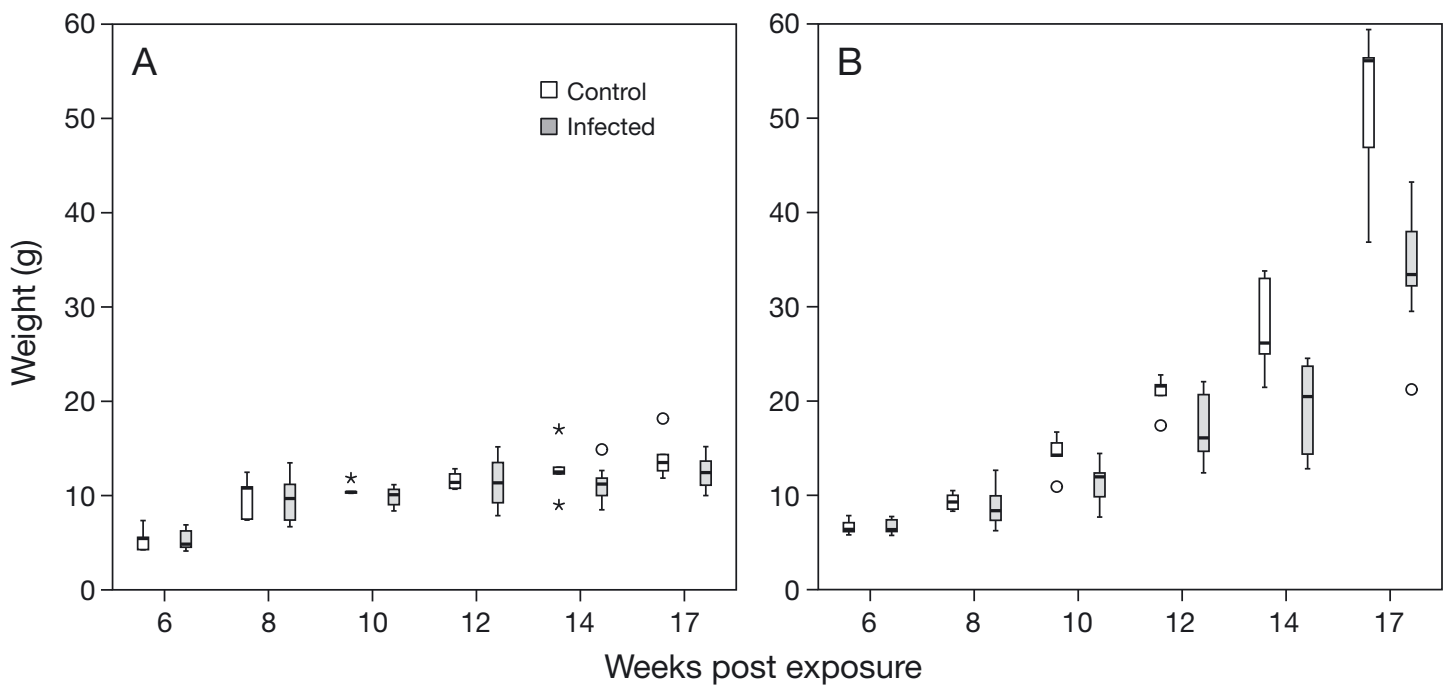

Fig. 2. Tetracapsuloides bryosalmonae infecting Salmo trutta and Oncorhynchus mykiss. Comparison of body weight of infected and control fish at different time points: (A) brown trout, (B) rainbow trout. Significant differences between control and infected fish were observed only in rainbow trout, beginning from $10 \mathrm{wk}$ post exposure. Boxplot details as in Fig. 1

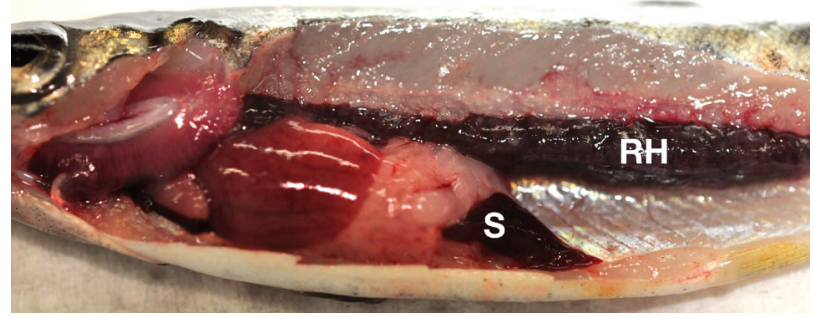

Fig. 3. Tetracapsuloides bryosalmonae infecting Salmo trutta. Brown trout showing clinical signs of proliferative kidney disease: renal hypertrophy (RH) and splenomegaly (S)
F. sultana colonies that cohabitated with infected rainbow trout showed no visible Tetracapsuloides bryosalmonae stages at any time up to 4 mo post exposure.

\section{qRT-PCR}

Parasite loads were significantly higher in kidneys of Tetracapsuloides bryosalmonae-infected brown trout compared with rainbow trout at all time points except at 6 wpe (Fig. 5). At 6 wpe, parasite load was 


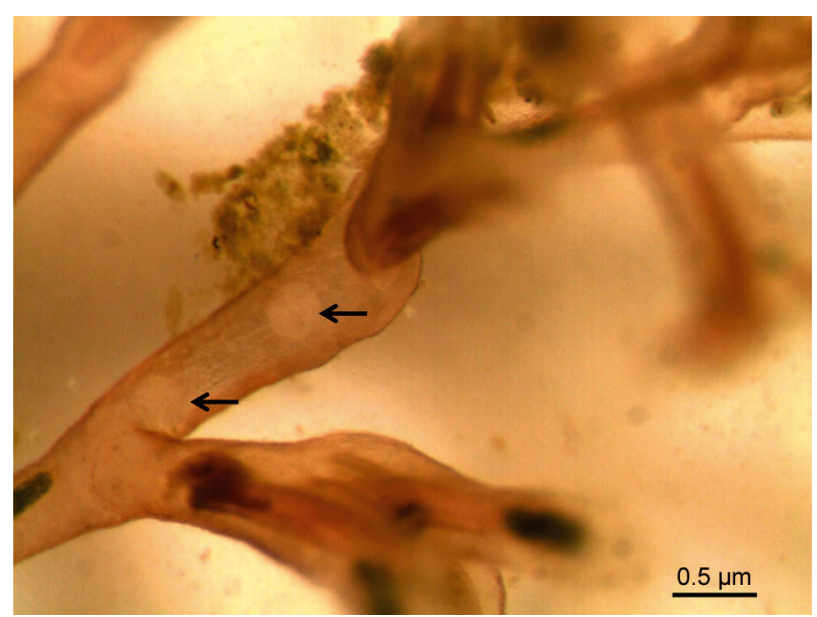

Fig. 4. Tetracapsuloides bryosalmonae infecting Fredericella sultana. Two spore sacs of the parasite (arrows) in the body cavity of $F$. sultana cohabitated with infected brown trout

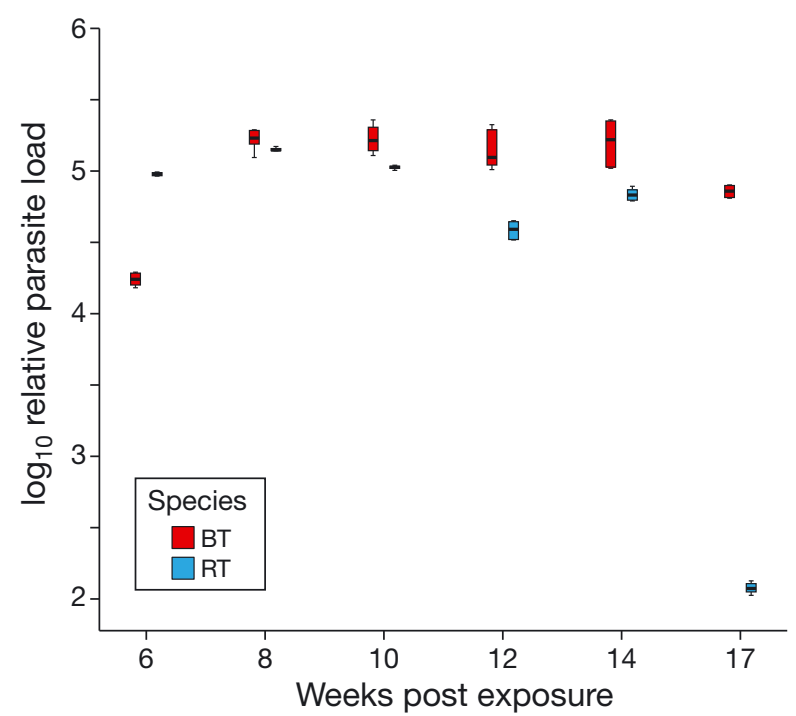

Fig. 5. Tetracapsuloides bryosalmonae infecting Salmo trutta and Oncorhynchus mykiss. Comparison of relative parasite load in kidneys of infected brown trout (BT) and infected rainbow trout (RT) at different time points. Normalized relative parasite load as determined by quantitative real-time PCR (qRT-PCR) in kidneys of infected brown trout and infected rainbow trout. The qRT-PCR data were $\log _{10}$-transformed and analyzed for significance at each time point. Boxplot details as in Fig. 1

significantly higher $(\mathrm{p}<0.001)$ in rainbow trout than in brown trout, while at 17 wpe, parasite load was almost undetectable in rainbow trout.

\section{Immunohistochemistry}

Kidney sections of brown trout and rainbow trout showed interstitial developmental stages of Tetra- capsuloides bryosalmonae (pre-sporogonic stages) and a proliferation of macrophages in interstitial tissue. The number of the interstitial pre-sporogonic parasite stages in kidneys was low in brown trout (Fig. 6A) but high in rainbow trout (Fig. 7A). Significant differences in the numbers of parasite stages were observed in brown trout compared with rainbow trout at 6, 8, and 14 wpe (Fig. 8). At 6 and 8 wpe, the number of parasite stages in rainbow trout was significantly higher $(\mathrm{p}<0.001)$ compared with brown trout. The differences in the number of parasite stages were not significant at 10 and 12 wpe $(p=0.885$, 0.418 , respectively). Overall, the number of parasite stages was higher in the kidneys of rainbow trout compared to brown trout at all time points. No parasite stages were detected in kidney sections of either brown trout or rainbow trout at 17 wpe.

High numbers of intra-luminal stages (sporogonic stages) of Tetracapsuloides bryosalmonae were detected in the renal tubules of brown trout. Sporogonic stages were detected in brown trout at 6 to 14 wpe, and renal tubes were filled with sporogonic stages at 8 to 12 wpe (Fig. 6B). Sporogonic stages were not seen in tubule lumens of rainbow trout but were observed occasionally in the tubular epithelial cells (Fig. 7B). Maturing spores with polar capsules were observed exclusively in renal tubules of brown trout.

Pre-sporogonic parasite stages were also detected in spleen and liver sections of brown trout and rainbow trout. At 6 wpe, few parasites were detected in the spleen of brown trout, and no parasite was detected in the liver, while in rainbow trout, parasite numbers were moderate in spleen and low in the liver. At 8 and 10 wpe, the numbers of parasite stages were low in the spleen (Fig. 6C) and liver of brown trout (Fig. 6D) and moderate in rainbow trout (Fig. $7 \mathrm{C}, \mathrm{D}$ ), while at 12 wpe, no parasite stages were seen in the spleen and liver of brown trout and low parasite numbers were seen only in the spleen of rainbow trout. No parasite stages were observed in the spleen and liver of brown trout at 14 and 17 wpe, but low parasite numbers were seen in the spleen and liver of rainbow trout at 12 and 14 wpe and none at 17 wpe.

\section{DISCUSSION}

In this study, we compared the development and intensity of Tetracapsuloides bryosalmonae in brown trout and rainbow trout from 6 to 17 wpe and transmission of $T$. bryosalmonae from infected fish to the bryozoan Fredericella sultana. No significant differ- 


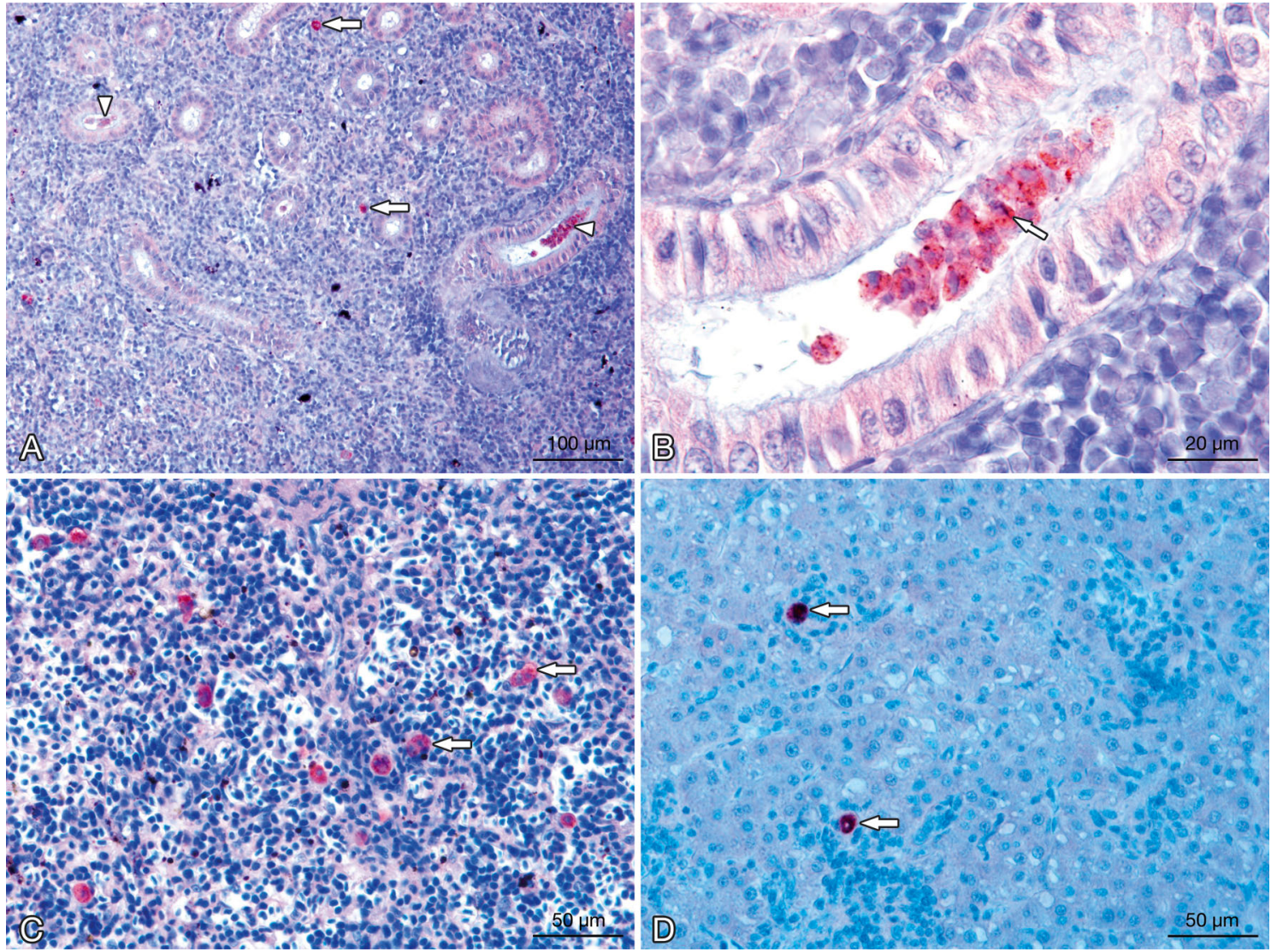

Fig. 6. Tetracapsuloides bryosalmonae infecting Salmo trutta. Stages in kidney, spleen, and liver tissues of infected brown trout. (A) Interstitial pre-sporogonic parasite stages (arrows), sporogonic stages (arrowheads), and proliferation of the interstitial tissue can be seen in the kidney. (B) Tubule lumen filled with numerous intraluminal sporogonic stages of the parasite. Polar capsules are visible (arrow). (C) Pre-sporogonic parasite stages in the spleen (arrows). (D) Parasite stages in the liver (arrows). All sections were stained using immunohistochemistry and a hematoxylin counter-stain

ence in the length and weight of infected brown trout was observed compared with controls, whereas significant differences were observed in length and weight of infected rainbow trout after 10 wpe (Fig. 1B). This result suggests that $T$. bryosalmonae influences the growth of rainbow trout but not brown trout after the onset of the clinical phase of infection.

Clinical signs of PKD were not observed in Tetracapsuloides bryosalmonae infected-brown trout during the investigation period (3-18 wpe) at water temperatures of 17.2 to $11.8^{\circ} \mathrm{C}$, although histopathological changes associated with infection were seen in the kidney (Clifton-Hadley \& Feist 1989). Similarly, none of the brown trout and rainbow trout showed clinical signs of PKD during 2 to 4 wpe at water temperatures of 14.4 to $15.4^{\circ} \mathrm{C}$ (Grabner \& ElMatbouli 2009). In a study by Holzer et al. (2006), minor to moderate swelling of kidneys and spleen were observed in only $12 \%$ of brown trout over a year, in fish exposed to pond waters in central Scot- land. Morris \& Adams (2008) observed slight kidney swelling in brown trout at 7 wpe to $T$. bryosalmonaeinfected Fredericella sultana colonies. We observed clinical signs of PKD such as anemia, renal hypertrophy, and splenomegaly in most of the brown trout $(90 \%)$ at 8 to 17 wpe. El-Matbouli et al. (2009) recorded clinical signs of PKD in German (Hofer strain) and North American (Troutlodge) rainbow trout at 4 to 16 wpe. They found renal hypertrophy and splenomegaly and pale liver in both strains. Furthermore, Schmidt-Posthaus et al. (2012) observed that kidneys of rainbow trout were heavily swollen at 6 wpe ( 47 dpe) with moderate swelling at 10 wpe (75 dpe); kidneys sampled 10 wpe were normal. We observed that kidneys of rainbow trout were intensely swollen at 8 to 12 wpe and moderately swollen at 14 wpe, but appeared normal at 17 wpe. Also, heavy enlargement of spleen and pale liver were observed in rainbow trout at 8 to 12 wpe, but they appeared normal at 17 wpe. 

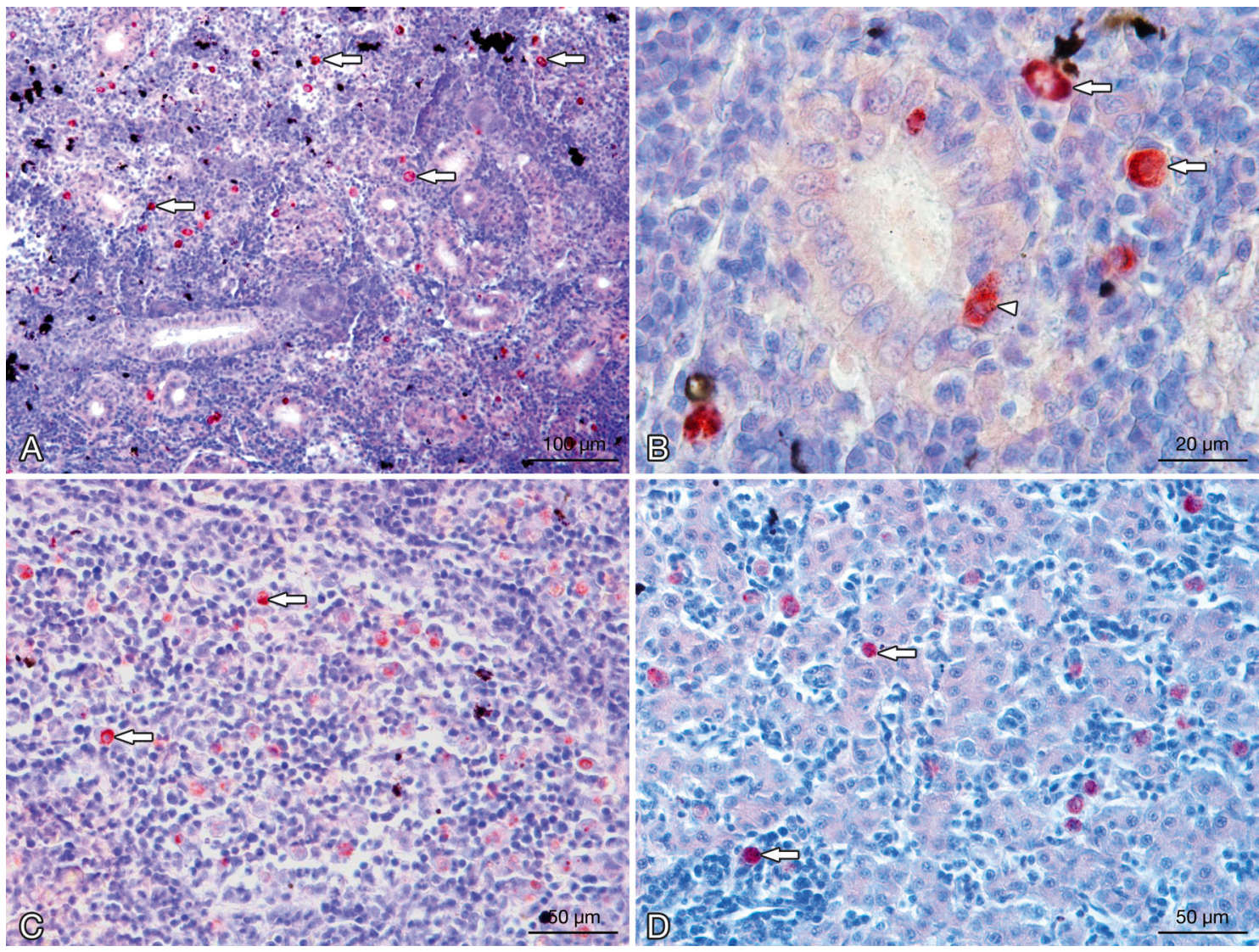

Fig. 7. Tetracapsuloides bryosalmonae infecting Oncorhynchus mykiss. Stages in kidney, spleen, and liver tissues of infected rainbow trout. (A) Numerous interstitial pre-sporogonic stages of the parasite (arrows); proliferation of the interstitial tissue can be seen in the kidney. (B) Interstitial stages in the kidney (arrows) and interepithelial stages of the parasite in kidney tubules (arrowhead). (C) Pre-sporogonic parasite stages in the spleen (arrows). (D) Parasite stages in the liver (arrows). All sections were stained using immunohistochemistry and a hematoxylin counter-stain

In studies conducted in Europe on rivers and farms where PKD occurred annually, mature spores formed in the kidney tubules of brown trout but not rainbow trout (Bucke et al. 1991). Significantly, in similar experiments in North America, rainbow trout formed spores in kidney tubules (Kent \& Hedrick 1986), but the prevalence of spores in the urine of rainbow trout was low over a year, after which no spores were observed (Hedrick et al. 2004). This led to the conclusion that there are 2 lineages of Tetracapsuloides bryosalmonae: one adapted to the genus Salmo and the other to the genus Oncorhynchus (Bucke et al. 1991, Morris et al. 1997). This conclusion is further supported by internal transcribed spacer sequence data (Henderson \& Okamura 2004), which resolve distinct European and North American lineages of T. bryosalmonae. Also, Grabner \& El-Matbouli (2008) showed that rainbow trout infected with the European $T$. bryosalmonae could not transmit the parasite to bry- ozoans, but brown trout could. A study conducted in Switzerland by Bettge et al. (2009a) demonstrated the presence of parasite DNA in urine of $T$. bryosalmonae-infected rainbow trout at 26 to 47 dpe, with no parasite DNA detected at 75 to 201 dpe (Schmidt-Posthaus et al. 2012). In their studies, no attempts were made to show whether this DNA was derived from developmental stages or just from cellular remains of parasite cells. Therefore, it is not clear whether the fish used in these experiments would have been able to transmit the parasite to bryozoans. Our study demonstrated again that rainbow trout infected with the European $T$. bryosalmonae were unable to infect Fredericella sultana colonies. Therefore, it can be assumed that formation of spores is a rare event in rainbow trout. Possible strain differences between parasites and or hosts from the USA and Europe were discussed by Morris \& Adams (2006), which could be confirmed in the present study. 


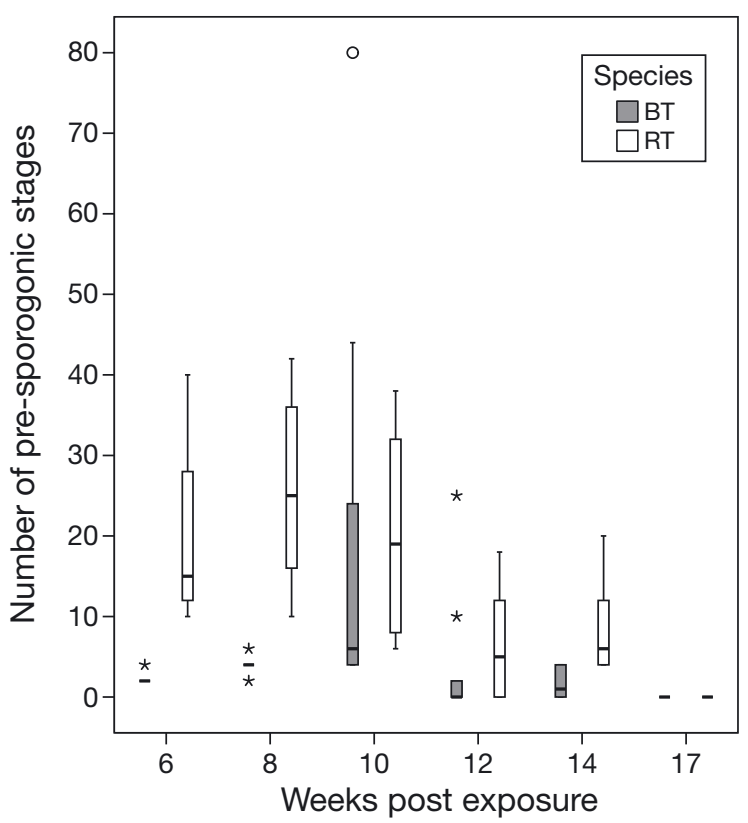

Fig. 8. Tetracapsuloides bryosalmonae infecting Salmo trutta and Oncorhynchus mykiss. Comparison of numbers of presporogonic parasite stages between infected brown trout (BT) and infected rainbow trout (RT) kidneys, detected using immunohistochemistry at different time points. Boxplot details as in Fig. 1

Schmidt-Posthaus et al. (2012) quantified temperature- and time-related Tetracapsuloides bryosalmonae DNA in the kidneys of rainbow trout kept at 12 and $18^{\circ} \mathrm{C}, 47$ to $201 \mathrm{dpe}$. They observed a gradual decline of parasite DNA after 12 wpe (103 dpi) at $18^{\circ} \mathrm{C}$, with DNA detected up to 18 wpe (131 dpe) at $18^{\circ} \mathrm{C}$ and up to 28 wpe $(201 \mathrm{dpe})$ at $12^{\circ} \mathrm{C}$. We found parasite DNA levels from 6 to 14 wpe in kidneys of rainbow trout kept at a constant water temperature $\left(16.5 \pm 1{ }^{\circ} \mathrm{C}\right)$, while DNA levels were almost undetectable at 17 wpe. This difference in the detection limit of parasite DNA may be due to an influence of water temperature on the development of $T$. bryosalmonae in rainbow trout (El-Matbouli \& Hoffmann 2002). The relationship between different water temperatures and the intensity of parasite infection was examined in rainbow trout, and it has been shown that the development and pathology of PKD are influenced by water temperature (Bettge et al. 2009a,b, Schmidt-Posthaus et al. 2012). In addition, our study demonstrated that brown trout had significantly higher parasite load compared to rainbow trout, confirming that brown trout are more susceptible to PKD.

The immunohistochemistry study of SchmidtPosthaus et al. (2012) showed that the number of parasite pre-sporogonic stages in the kidneys of rainbow trout declined between 6 and 10 wpe (47 and 75 dpe) and after that, no parasites were detected. In our study, the numbers of pre-sporogonic stages were low in kidneys of brown trout and high in the kidneys of rainbow trout. In rainbow trout, numbers declined after 10 wpe and were undetectable after 14 wpe. In brown trout, the number of pre-sporogonic stages was very low in kidneys of brown trout at 6 wpe, increased at 8 to 10 wpe, then decreased between 12 and 14 wpe to be undetectable after that. CliftonHadley \& Feist (1989) examined the pre-sporogonic stages in the kidneys of brown trout at 5, 7, 9, and 18 wpe and sporogonic stages at 9 or 14 to 18 wpe. Holzer et al. (2006) observed pre-sporogonic stages at 17 to 36 wpe and sporogonic stages at 30 to 45 wpe in brown trout. Few pre-sporogonic stages were detected in kidneys of brown trout and rainbow trout at 2 to 4 wpe (Grabner \& El-Matbouli 2009). Parasite cells were found in the kidney interstitium at 47 to 75 dpe and sporogonic stages at 47 dpe in rainbow trout (Schmidt-Posthaus et al. 2012). However, in the present study, we observed interstitial pre-sporogonic stages at 6 to 14 wpe in both fish species. Moreover, the renal tubules of brown trout were filled with sporogonic stages of the parasite (Fig. 6B), whereas in rainbow trout, occasionally parasites were found interepithelial in the kidney tubules (Fig. 7B). Our results confirm that the development and distribution of the parasite in brown trout and rainbow trout vary at different time points of infection.

In other organs, Clifton-Hadley \& Feist (1989) observed the 'PKX' cells in the spleen of brown trout at 5 to 18 wpe, but not in the liver. Grabner \& ElMatbouli (2009) reported that no parasite was detected in the spleen and liver of most brown and rainbow trout at 2 to 4 wpe. Holzer et al. (2006) observed pre-sporogonic stages in spleen and liver of brown trout at 17 to 36 wpe. In the histological analysis performed by El-Matbouli et al. (2009), the numbers of parasites in the spleen were low for both German and North American rainbow trout strains, with no stages observed in the liver throughout the investigation (4 to 16 wpe). However, in the present study, we observed moderate numbers of pre-sporogonic stages in both spleen and liver of brown trout at 8 to 10 wpe and in rainbow trout at 6 to 10 wpe using immunohistochemistry.

The macrophage is the main cell type involved in the inflammatory response to Tetracapsuloides bryosalmonae infection, and it has been suggested that the macrophages can stop development of $T$. bryosalmonae and eliminate the parasite from the host (MacConnell et al. 1989). Further, it has been sug- 
gested by flow cytometry that lymphocytes play an important role in proliferation of lymphoid tissues of fish, contributing to the clinical signs of PKD (Chilmonczyk et al. 2002). Thus, it was assumed that the innate immunity of the host participates in the reaction against the parasite. In the present study, both qRT-PCR and immunohistology studies showed remarkable differences in parasite quantity, numbers, and developmental stages between brown trout and rainbow trout and also in transmission of $T$. bryosalmonae from infected fish to the bryozoan. These differences prove that both of the fish species express different defense mechanisms in response to the development and distribution of the parasite. Our data support previous studies that the differences observed between brown trout and rainbow trout are caused by differences in the response of their innate immune systems to $T$. bryosalmonae (Klontz et al. 1986, MacConnell et al. 1989, Chilmonczyk et al. 2002).

The difference of Tetracapsuloides bryosalmonae development and its infection intensity in brown trout and rainbow trout examined chronologically in this study opens the door for further genetic analyses of host immune genes. Further work is in progress to characterize the genes responsible for the successful sporogenesis of $T$. bryosalmonae in brown trout and not in rainbow trout at a genetic level using a suppression subtractive hybridization technique.

Acknowledgements. This study was funded by the Austrian Science Fund (FWF) project no. P 22770-B17. We thank A. Tichy for statistical data analysis and B. Eckel for assisting with fish maintenance.

\section{LITERATURE CITED}

Bettge K, Segner H, Burki R, Schmidt-Posthaus H, Wahli T (2009a) Proliferative kidney disease (PKD) of rainbow trout: temperature- and time-related changes of Tetracapsuloides bryosalmonae DNA in the kidney. Parasitology 136:615-625

Bettge K, Wahli T, Segner H, Schmidt-Posthaus H (2009b) Proliferative kidney disease in rainbow trout: time- and temperature-related renal pathology and parasite distribution. Dis Aquat Org 83:67-76

$>$ Bucke D, Feist SW, Clifton-Hadley RS (1991) The occurrence of proliferative kidney disease (PKD) in cultured and wild fish: further investigations. J Fish Dis 14: 583-588

Chilmonczyk S, Monge D, De Kinkelin P (2002) Proliferative kidney disease: cellular aspects of the rainbow trout, Oncorhynchus mykiss (Walbaum), response to parasitic infection. J Fish Dis 25:217-226

Clifton-Hadley RS, Bucke D, Richards RH (1987) A study of the sequential clinical and pathological changes during proliferative kidney disease in rainbow trout, Salmo gairdneri Richardson. J Fish Dis 10:335-352

El-Matbouli M, Hoffmann RW (2002) Influence of water quality on the outbreak of proliferative kidney diseasefield studies and exposure experiments. J Fish Dis 25: 459-467

El-Matbouli M, Mattes M, Soliman H (2009) Susceptibility of whirling disease (WD) resistance and WD susceptible strains of rainbow trout Oncorhynchus mykiss to Tetracapsuloides bryosalmonae, Yersinia ruckeri and viral haemorrhagic septicaemia virus. Aquaculture 288: 299-304

Feist SW, Longshaw M, Canning EU, Okamura B (2001) Induction of proliferative kidney disease (PKD) in rainbow trout Oncorhynchus mykiss via the bryozoan Fredericella sultana infected with Tetracapsula bryosalmonae. Dis Aquat Org 45:61-68

Ferguson HW, Needham EA (1978) Proliferative kidney disease in rainbow trout Salmo gairdneri Richardson. J Fish Dis 1:91-108

Grabner DS, El-Matbouli M (2008) Transmission of Tetracapsuloides bryosalmonae (Myxozoa: Malacosporea) to Fredericella sultana (Bryozoa: Phylactolaemata) by various fish species. Dis Aquat Org 79:133-139

Grabner DS, El-Matbouli M (2009) Comparison of the susceptibility of brown trout (Salmo trutta) and four rainbow trout (Oncorhynchus mykiss) strains to the myxozoan Tetracapsuloides bryosalmonae, the causative agent of proliferative kidney disease (PKD). Vet Parasitol 165: 200-206

Hedrick RP, Baxa DV, de Kinkelin P, Okamura B (2004) Malacosporean-like spores in urine of rainbow trout react with antibody and DNA probes to Tetracapsuloides bryosalmonae. Parasitol Res 92:81-88

> Henderson M, Okamura B (2004) The phylogeography of salmonid proliferative kidney disease in Europe and North America. Proc R Soc Lond B Biol Sci 271: 1729-1736

Holzer AS, Sommerville C, Wootten R (2006) Molecular studies on the seasonal occurrence and development of five myxozoans in farmed Salmo trutta L. Parasitology 132:193-205

- Kent ML, Hedrick RP (1986) Development of the PKX myxosporean in rainbow trout Salmo gairdneri. Dis Aquat Org 1:169-182

Klontz GW, Rourke AW, Eckblad W (1986) The immune response during proliferative kidney disease in rainbow trout: a case history. Vet Immunol Immunopathol 12: 387-393

> Kumar G, Abd-Elfattah A, Soliman H, El-Matbouli M (2013) Establishment of medium for laboratory cultivation and maintenance of Fredericella sultana for in vivo experiments with Tetracapsuloides bryosalmonae (Myxozoa). J Fish Dis 36:81-88

- MacConnell E, Smith CE, Hedrick RP, Speer CA (1989) Cellular inflammatory response of rainbow trout to the protozoan parasite that causes proliferative kidney disease. J Aquat Anim Health 1:108-118

Morris DJ, Adams A (2006) Transmission of Tetracapsuloides bryosalmonae (Myxozoa: Malacosporea), the causative organism of salmonid proliferative kidney disease, to the freshwater bryozoan Fredericella sultana. Parasitology 133:701-709

Morris DJ, Adams A (2008) Sporogony of Tetracapsuloides bryosalmonae in the brown trout Salmo trutta and the 
role of the tertiary cell during the vertebrate phase of myxozoan life cycles. Parasitology 135:1075-1092

Morris DJ, Adams A, Richards RH (1997) Studies of the PKX parasite in rainbow trout via immunohistochemistry and immunogold electron microscopy. J Aquat Anim Health 9:265-273

Morris DJ, Ferguson HW, Adams A (2005) Severe, chronic proliferative kidney disease (PKD) induced in rainbow trout Oncorhynchus mykiss held at a constant $18^{\circ} \mathrm{C}$. Dis Aquat Org 66:221-226

Okamura B, Hartikainen H, Schmidt-Posthaus H, Wahli T

Editorial responsibility: David Marcogliese,

Montreal, Quebec, Canada
(2011) Life cycle complexity, environmental change and the emerging status of salmonid proliferative kidney disease. Freshw Biol 56:735-753

Schmidt-Posthaus H, Bettge K, Forster U, Segner H, Wahli T (2012) Kidney pathology and parasite intensity in rainbow trout Oncorhynchus mykiss surviving proliferative kidney disease: time course and influence of temperature. Dis Aquat Org 97:207-218

Tops S, Baxa DV, McDowell TS, Hedrick RP, Okamura B (2004) Evaluation of malacosporean life cycles through transmission studies. Dis Aquat Org 60:109-121

Submitted: May 21, 2013; Accepted: August 29, 2013 Proofs received from author(s): October 31, 2013 